\title{
The Influence of Pre-training Factors on Motivation to Transfer Learning at the Post Training Stage
}

\author{
Bekolo Ngoa Celestin \\ School of Management and Economic, University of Electronic Science and Technology of \\ China \\ PO Box: No. 2006, Xiyuan Ave. West Hi-Tech. Zone, P. R. China \\ Tel: 86-182-8034-4576Ｅ-mail: bekolo.celestin@gmail.com
}

\begin{abstract}
Prof Shao Yufen
School of Management and Economic, University of Electronic Science and Technology of China

PO Box: No. 2006, Xiyuan Ave. West Hi-Tech. Zone, P. R. China

Tel: 86-134-0847-9937Ｅ-mail: shaoyf@uestc.edu.cn
\end{abstract}

Received: January 14, 2018 Accepted: March 20, 2018 Published: March 22, 2018

doi: 10.5296/hrr.v2i1.12483 URL: https://doi.org/10.5296/hrr.v2i1.12483

\begin{abstract}
Transfer of training is the ultimate aim of training investment and the key to maintain competitive advantage in today's rapidly changing operating environment where organizational success often depends on the motivation with which employee can learn and apply new ideas and information. While previous researches have focused on factors at the training stage influencing motivation to transfer training at the post-training stage, this study investigates the influence of pre-training factors. Particularly, pre-training performance self-efficacy, learning readiness, perceived content validity, and organization openness to change. The result should assist managers and trainers to ascertain the trainee state of preparedness before the training program to anticipate successful transfer of learning from the workshop to the workplace. A survey of high and vocational school teachers in Thailand participating in 5 days training on cloud computing integration in teaching was made using the Learning Transfer Inventory System (LTIS). Results show that learning readiness, perceived content validity, and organization openness to change influence the motivation to
\end{abstract}


transfer at the post-training stage. Thus, framing the training program in the way trainees can answer to the questions "can I do this task?", "do I want to do this task and why?" at the pre-training stage influence motivation to transfer. An implication to managers is that employees' selection for training should take into consideration trainees' perceptions in order to anticipate motivation to transfer at the post-training stage.

Keywords: Motivation to transfer, Learning readiness, Performance self-efficacy, Perceived content validity, Organization openness to Change

\section{Introduction}

Little empirical research has been carried out to understand the influence effect of pre-training factors on training outcomes (Bell, Tannenbaum, Ford, Noe, \& Kraiger, 2017). Although some research have paid attention to training intervention and professional development stages (Grohmann \& Kauffeld, 2013; Korthagen, 2017; Rotermund, Deroche, Ottem, Owens, \& O 'rear, 2017), little is known to what extent pre- training factors account for variance in motivation to transfer learning at the post-training stage. In addition, before the training program, trainees general faced the questions "can I do this task?", "do I want to do this task and why?", and "what do I have to do to succeed in the task?" Few studies have explored how answers to these questions influence motivation to transfer skill at the post-training stage. Furthermore, Gegenfurtner, Veermans, Festner, and Gruber (2009) hypothesized that at the pre-training stage, long before the training program, trainees may be motivated or not to transfer what they are going to learn on the job, depending on pre-training individual attitudes and attributes. However, this assertion lacks empirical evidence.

A major point of difference between the trainee at the pre-training stage and post-training stage is the change in knowledge or skill acquired during the training program. At the post-training stage the motivation to transfer is led by the "desire to use the knowledge and the skills mastered in the training program on the job" (Noe \& Schmitt, 1986, p. 743) which is antecedent to the same trainees' motivation to learn the training content before they attend a training program (Devos et al., 2007; Edwards, 2013). In addition, as individual characteristics such as self-efficacy are more or less stable and cannot be significantly changed during the training program, researches have concluded that self-efficacy at the pre-training stage is a predictor of self-efficacy at the post training stage supporting the fact that, some of the trainee characteristics at the pre-training stage do not change at the posttraining stage (Chung, 2013).

The influence of pre-training factors on the motivation to transfer training at the post-training stage lacks empirical evidence. Researchers have suggested several steps to follow at the pre-training stage to anticipate a successful training transfer. However, very few of these suggestions have been empirically tested. As a result, there is no clear evidence as of which factors at the pre-training stage account for significant variance to the motivation to transfer training at the work place. For instance, Prasertsilp and Olfman (2014) suggest that at the pre-training stage, trainers should: (1) identify trainees needs and set goals, (2) develop training method, (3) select trainees and set in groups, (4) create training motivation, and (5) design required technology for training. Chung (2013) proposed that in order to anticipate the 
successful transfer of training from the workshop to the workplace, trainees must show three dimensions of readiness at the pre-training stage including, motivational readiness, behavioral readiness, and cognitive readiness. The assumption here is that, the trainee learning characteristics at the pre-training stage are equally important at the post-training stage.

As the transfer problem has been widely recognized in Professional Development (PD) where researches and practitioners consistently conclude that the return on many training investments is low and organizational investments in training are too often wasted, a new perspectives seems to point out that the transfer problem is not only associated to a lack of adequate training but also to a lack of understanding trainee's characteristics at the pre-training stage (Chang \& Chiang, 2013). In an attempt to address this transfer problem from the abovementioned new perspective, this study focuses on the pre-training factors that may have a significant influence on the motivation to transfer learning from a PD intervention. Specifically this paper addresses the transfer of training in cloud computing among secondary and vocational schools teachers in Thailand. The leading questions of this study centers on how trainees answers to the questions: (1) can I do this task? (Learner readiness, performance self-efficacy), (2) do I want to do this task and why? (perceived content validity), and (3) how does organization openness to change account for variance in motivation to transfer training content at the work place?

This research aims at empirically testing how pre-training factors influence post- training motivation to transfer by measuring the amount of variance pre-training factors account for change in motivation to transfer. That is, to identify key factors at the pre-training stage and links them to motivation to transfer. Essentially, we respond to a call for new thinking about PD intervention and draws inspiration from Chang and Chiang (2013) who has stressed the need to understand trainees individual factors at the work place and organization normative set up prior to the training program in order to anticipate transfer .

Findings from this study provides administrators, and trainers with a better understanding of individual and organizational factors tie with the transfer problem before the training program. Managers can use the findings in this study to understand how trainee characteristics interacts with program design features to affect learning outcomes. Trainer providers can build on pre-training factors to customized training strategy according to learners' characteristics.

The rest of the paper is structured as follow. First, a conceptual framework explaining the effect of pre-training factors on motivation to transfer is presented. Next, the procedure used to test the hypothesized effect of pre-training factors on motivation to transfer is described. The findings of the study are then presented. The article concludes with a summary of the study's contribution to research and directions for future research.

\section{Literature Review}

Noe and Schmitt (1986), were the first to introduce the construct motivation to transfer (Transfer motivation is a synonym) and defined it as "the trainees desire to use the knowledge and the skills mastered in the training program on the job" (p. 743). Furthermore, they suggested that motivation to transfer is affected by environmental favorability. Since then, 


\section{Macrothink

researches have focused on how transfer motivation at the post-training stage is affected by individual characteristics. Specifically, researches have addressed the relations of transfer motivation to attitudes toward training, motivation to learn, personality traits, and work commitment (Banerjee, Gupta, \& Bates, 2017; Chang \& Chiang, 2013). However, these studies report divergent effect sizes and inconsistencies.

Building on Broussard (2004) observation of the contemporary focus of the motivation research, pre-training factors affecting motivation to transfer learning at the post-training stage might be found in the trainees' answers of the following questions before the training program:

- Can I do this task?

- Do I want to do this task and why?

In addition, we posit that at the pre-training stage, the organizational normative context already functions to promote or hinder the development of transfer motivation. Using Figure 1 as a framework, components and contributions are discussed in turn.

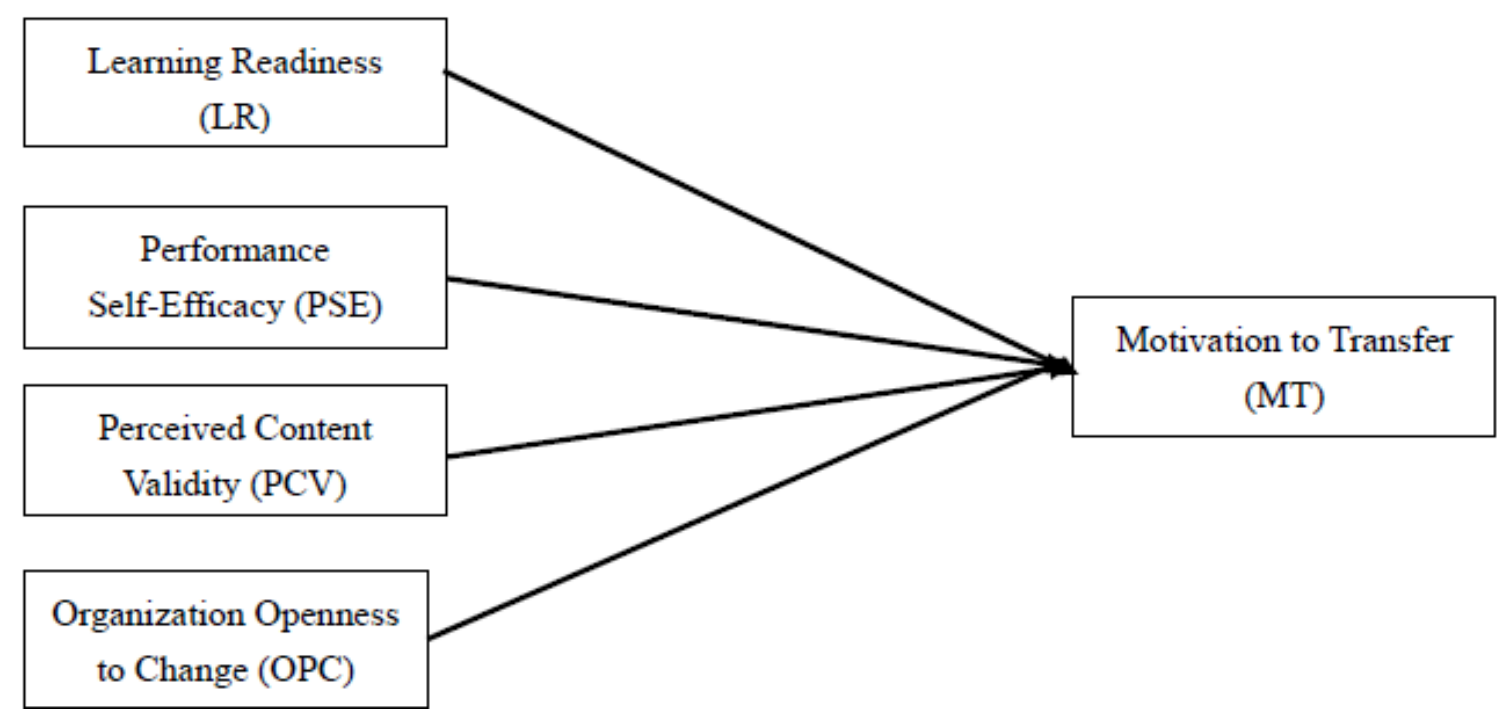

Figure 1. Conceptual framework of pre-training factors influence on motivation to transfer

\subsection{Can I Do This Task?}

The answer of the above question which influences the motivation to transfer at the post-training stage is supported by self-efficacy theory and attribution theory. Pre-training self-efficacy is defined as the degree of trainees' beliefs at the pre-training stage that they can change their performance when they want (Chang \& Chiang, 2013; Reid Bates, Holton, \& Paul, 2012). If trainees believe they can acquire and retain knowledge and skills from a training program (high pre-training self-efficacy), they will devote efforts to learning (Bandura, 2009). In the literature, evidence show a positive effect of pre-training self-efficacy on training outcome (Chiaburu \& Marinova, 2005; Chang \& Chiang, 2013; De Rijdt et al., 
2013; Forehand et al., 2017). High self-efficacy trainee are likely to be motivated to learn training content (Bozdoğan \& Özen, 2014), enhance learning and utility of training, and improve job performance (Kao, Tsai, \& Shih, 2014). In addition, trainees with high self-efficacy to learn training content also anticipate that effort devoted to transferring learning in the workplace will lead to changes in job performance. Therefore, it is posited that:

H1: Pre- Training performance self-efficacy account for significant amount of variance in motivation to transfer learning at the work place.

Another aspect to answer the question "can I do this task?" at the pre-training stage depends on the locus of control. According to this theory, individuals would be motivated when they feel that they are in control of their own success and failure. Locus control is linked to the concept of self-attributions. Self-attributions is defined as an individual's belief of being in control of the causes of successful or failing performance. An individual can have several types of attributions, including ability, effort, task, and luck. According to the attribution theory, the types of attributions a person holds determine his or her level of motivation (Broussard, 2004).

In the Learning Transfer Inventory System (LTIS), Holton (2005) hypothesized that, individual perceived learning readiness which closely related to individual attribution affects learning and transfer of training. Learning readiness is defined as "the extent to which individuals are prepared to enter and participate in training" (Devos et al., 2007). Leaning readiness is supported in the literature, as an influencing factor to motivation to transfer and ultimately a significant predictor of effective transfer of the training content at the work place (De Rijdt et al., 2013; Kontoghiorghes, 2002; Kirwan \& Birchall, 2006). Findings indicate correlation coefficients between learning readiness and motivation to transfer raging between .33 and .75 (De Rijdt et al., 2013). Leaner readiness denote trainee motivational factors. It examines the level of preparedness both physically and psychologically of individual trainee prior to entering training (Ruona, Iii, \& Bates, 2002). Reid, Bates et al., (2007) argued the learner readiness demonstrates trainee (a) understanding of a program that affects the performance, (b) understanding about job related developments, (c) expectations from training, and (d) expected outcomes at the beginning of the training (Devos et al., 2007). Based on the aforementioned discussion, it is hypothesized that:

H2: Pre-training learning readiness accounts for significant amount of variance in motivation to transfer learning at the work place.

\subsection{Do I Want to Do This Task and Why?}

The second most important question trainees must answer before attending training program in order to anticipate positive motivation to transfer at the post-training stage is "Do I want to do this task and why ?" The answer to this question includes a combination of expectancy-value theories, intrinsic motivation theories, and self-determination theory.

One strand of this answer is based on the value individual's hold for participating in various type of activities including training. Values are incentives or reason for participating in an 
activity (Appova \& Arbaugh, 2017). The value of a given task or activity has four components: attainment value, which refers to a personal value of doing well on the task; Intrinsic value, which refers to subjective interest or enjoyment of performing a task; Utility value which refer to the extent to which task completion is perceived to facilitate current or future goals; and Cost, which refers to the negative aspects of engaging in a given task, such as anxiety and fear of failure (Han, Yin, \& Boylan, 2016)

Perceived utility of training contents refers to a trainee's belief or opinion that the training program content is to be useful for his/her job (Chang \& Chiang, 2013). If trainees perceive that the content of training program is useful, they will be more likely to use or apply the knowledge and skills learned from the training program to their jobs. They develop a high motivation to transfer training content. Before the training program, trainees evaluate the training content to see if the training program has practical value. Pre-training perceived utility is reflected on the motivation to transfer training at the post-training stage as well as motivation to learn. Bates and Holton (2004) and Tai (2006) suggested that, communicating the company's expectations before the training program which also promotes learner readiness leads to enhanced transfer motivation. The detail level in which the training program is framed before the training event determines the extent a trainee is motivated to transfer learning to the workplace (Appova \& Arbaugh, 2017). In LTSI, perceived utility of training, content is referred by perceived content validity which is the extent to which trainee's judge training content to accurately reflect job requirements. It is therefore posited that:

H3: Perceived content validity of the training accounts for significant amount of variance in motivation to transfer learning at the work place

\subsection{Organization Normative Context and Motivation to Transfer}

Trainees are influenced by their organization norms even before they attend the training event. Previous researches on the effects of pre-training work environment on transfer motivation have focused primarily on organizational culture. Various levels of organization culture have been identified as antecedent of training success and transfer motivation (Zubairy, Mozie, \& Ghazali, 2015). Organization learning culture which reflects the values and beliefs about the importance of learning at work has been found to be positively related to trainee's transfer motivation (Zubairy et al., 2015). Kontoghiorghes (2002) shows that transfer motivation is high when trainees understand that they are accountable for the training application, that is, when the organization expects trainees to use the training in the workplace. Thus, before the training program even started, the organization normative context already functions to promote or hinder the development of transfer motivation. Therefore we posited that:

H4: The organization openness to change accounts for a significant amount of variance in motivation to transfer learning at the work place. 


\section{Methodology}

\subsection{Sampling}

The target population for this study consisted of Thais' teachers in vocational and high schools participating in 5 days Google Apps For Education (GAFE) training during the end of the academic year 2016/2017. GAFE is a set of productivity applications that Google put to schools and education institutions disposal for free. These communication and collaboration apps include Gmail, Calendar, Drive, Docs, Google classroom and Sites, and a G Suite for education account that opened access to dozens of other collaborative tools supported by Google (Iftakhar, 2016). All these applications exist completely online (or in the cloud), meaning that all apps can be accessed from any device with an Internet connection. Once a school decides to adopt the G Suite for Education, they can register their school domain, and administer all teachers and students account from an administrative dashboard. Because these applications are quite new to the teachers in Thailand, a series of trainings were organized to initiate the teacher in how to use these cloud applications.

The unit of analysis was the individual teacher. A list of 667 teachers generated from the online registration for training served as sampling frame. To account for the impact of low response rate normally associated with online surveys, the teachers were asked to answer the survey's questions from the very first day of the training. This was done in order to realize a large enough sample for the use of regression analysis. The final realized sample included a total of 232 usable questionnaires representing 34\% response rate. Only 213 questionnaires were analyzed.

The sample was slightly dominated by female respondents $54.9 \%$ against $45.1 \%$ male. With respect to age, $6.1 \%$ were lesser than 26 years old, $43.2 \%$ were between $26-35$ years old, $29.6 \%$ were $36-45$, and $15 \%$ were $46-55$ and $5.61 \%$ were over 55 years of age.

\subsection{Data Collection}

The initial questionnaire was pre-tested with a convenience sample of 15 teachers using the collaborative participant pre-testing method described by Cooper and Schindler (2006: 396). Since the researcher was part of the training team, he had a discussion with the other colleagues about the clarity in meaning of each question in the survey. No particular modification was suggested except the fact that some thought the survey questionnaire was too long. Data for the main study, were collected at four training location points designated by the Office of Basic Education (OBEC) Thailand since the training could not be held in every school. Training point locations were appointed to gather teachers from the surrounding schools. Specifically, for the training held at Chagcheasao vocational college (Central part of Thailand), 78 teachers participated in the survey, at Nakhorn Nayork Technical College (East of Thailand) 64, at Mathayom Wainarong School (Bangkok Metropolitan) 69 and at Sunthorn Phu Pitiya 21 respondents.

At the beginning of the training session, the researcher engaged the trainees (teachers) in an informal conversation about their reasons of attending the training, their expectations, and importance of such training. Participants were also told that at the end of the training they 
will be asked to answer the survey questions related to the training. The explanation of anticipating questions in the survey in an informal conversation was to prepare the trainees for the survey by giving them the time to really think about the answer. At the end of each training session, the trainees were directed to the online questionnaire, which was designed to assess the various variables in this study. While the participants were answering the questions, the researcher was present in the room to provide any clarification if needed. Additional, qualitative information was also obtained through focus groups with the trainers and interviews with other stakeholders (e.g. college administrators, principals and IT support staff) as part of the larger pilot project associated with this study. However, these data were used solely to inform our interpretation of the results of study analyses.

\subsection{Measures}

Learning Transfer Inventory System (LTSI) questionnaire version 3 developed by Reid Bates et al., (2012) was used to assess the constructs in this study. LTSI questions are constructed using a Likert-type scale ranging from 1 (Strongly Disagree) to 5 (Strongly Agree). The instrument is divided into two construct domains. The first section is training event specific and assesses an individual's perception attending a training program. This section measures eleven constructs including Learner Readiness (LR), Motivation to Transfer (MT), Positive Personal Outcomes (PPO), Negative Personal Outcomes (NPO), and Personal Capacity for Transfer (PCT), as well as Peer Support (PS), Supervisor Support (SS), Supervisor Sanctions (SST), Perceived Content Validity (PCV), Transfer Design (TD), and Opportunity to Use $(\mathrm{OU})$. The second domain examines training from a general organizational perspective and relates to training beyond a specific training event or session and evaluates a training program. This portion contains questions built to measure five constructs of transfer including Transfer Effort Performance Expectancy (TEPE), Expectation Performance Outcomes (EPO), Openness to Change(OP), Performance Self-Efficacy (PSE), and Performance Coaching (PC) (Iii, Bates, Bookter, \& Yamkovenko, 1996). From the study of Chang and Chiang (2013) these constructs were rearranged as of constructs at the pre- training stage, Training stage and post training stage. Table 1 is derived from an extraction from the matrix of LTSI training stages and training components proposed by Chang and Chiang (2013) with inclusion of the cronbach's Alpha of the constructs of interest in this study. 
Table 1. LTSI pre-training constructs definitions extracted from Chang and Chiang (2013)

\begin{tabular}{|c|c|c|c|c|c|}
\hline $\mathrm{N}$ & $\begin{array}{l}\text { Pre-training } \\
\text { constructs }\end{array}$ & Definition & Item Example & Questions & $\begin{array}{l}\text { Crombah } \\
\text { Alpha }\end{array}$ \\
\hline 1 & $\begin{array}{l}\text { Learner } \\
\text { readiness } \\
(L R)\end{array}$ & $\begin{array}{l}\text { The extent to which } \\
\text { individuals } \\
\text { prepared to enter and } \\
\text { participate in training. }\end{array}$ & $\begin{array}{l}\text { Before the training } \\
\text { I had a good } \\
\text { understanding of } \\
\text { how it would fit my } \\
\text { job related } \\
\text { development }\end{array}$ & $1,8,9$ & $\alpha=.818$ \\
\hline 2 & $\begin{array}{l}\text { Motivation to } \\
\text { Transfer } \\
(M T)\end{array}$ & $\begin{array}{l}\text { The direction, intensity } \\
\text { and persistence of } \\
\text { effort } \\
\text { toward utilizing in a } \\
\text { work setting skills and } \\
\text { knowledge learned }\end{array}$ & $\begin{array}{l}\text { I get excited when I } \\
\text { think about trying } \\
\text { to use my new } \\
\text { learning on my job. }\end{array}$ & $2,3,4$ & $\alpha=.879$ \\
\hline 3 & $\begin{array}{l}\text { Performance } \\
\text { self-efficacy } \\
(P S E)\end{array}$ & $\begin{array}{l}\text { An individual's } \\
\text { general belief that he is } \\
\text { able to change his } \\
\text { performance when he } \\
\text { wants to. }\end{array}$ & $\begin{array}{l}\text { I am confident in } \\
\text { my } \\
\text { ability to use newly } \\
\text { learned skills on } \\
\text { the job }\end{array}$ & $45,46,47$ & $\alpha=.818$ \\
\hline 4 & $\begin{array}{l}\text { Perceived } \\
\text { content } \\
\text { validity }(\mathrm{CV}))\end{array}$ & $\begin{array}{l}\text { The extent to which } \\
\text { trainee's judge training } \\
\text { content to reflect job } \\
\text { requirements } \\
\text { accurately }\end{array}$ & $\begin{array}{l}\text { What is taught in } \\
\text { training closely } \\
\text { matches my job } \\
\text { requirements }\end{array}$ & $27,28,29$ & $\alpha=.868$ \\
\hline 5 & $\begin{array}{l}\text { Organization } \\
\text { Openness to } \\
\text { Change } \\
(\text { OPC) }\end{array}$ & $\begin{array}{l}\text { Extent to which } \\
\text { prevailing group } \\
\text { norms are perceived by } \\
\text { trainees' to resist or } \\
\text { discourage the use of } \\
\text { skills and knowledge } \\
\text { acquired in training }\end{array}$ & $\begin{array}{l}\text { People in my group } \\
\text { are } \\
\text { open to changing } \\
\text { the } \\
\text { way they do things }\end{array}$ & 41,42 & $\alpha=.856$ \\
\hline
\end{tabular}

\subsection{Analysis}

To help answer the research questions relating to the predictors of motivation to transfer at the pre-training stage, multiple regressions analysis procedures seemed appropriate because this methodology helps to estimate the amount of unique variance a predictors account for in the dependent variable. Also, multiple regression help analyze the hypothesized model structure. In the context of this study, it specifically aimed at testing postulated casual effect system comprising the latent variable of Learning Readiness (LR), Performance Self-Efficacy 


\section{MInstitute Macrothink $_{\text {Int }}$}

(PSE), Personal Capacity for Transfer (PCT), Perceived Training Content Validity (PCV), Organization openness to Change (OC) and Motivation to Transfer (MT).

Analysis was conducted in four stages using SPSS 21. First data were screened to test for multivariate outliers' normality and multi-collinearity. Second, Cronbach Alpha was used to measure internal consistency among the measured variable. The result were validated at $\alpha>7$ (see table 1) which prove high internal consistency among variable within the construct. Third, we operated measure of central tendency by calculating the Mean and standard deviation of the sample data. Fourth, once the measurement models were established, the raw matrix was fitted to the hypothesized model to test the linear relationship among the constructs. Given evidence of inadequate fit, the model was modified by deleting non-significant parameters identified in ANOVA table and t-test respectively.

\section{Results}

\subsection{Preliminary Data Analysis}

Table 2 represents mean, Standard deviation, and inter-correlation for all constructs generated from EFA that, when taken together, explain 56.7 percent of the total variance in the data set. In testing for multivariate outlying case and unengaged respondents, no outlier was found and the standard deviation among responses of individual respondents were between .37 to 1.57 meaning that all respondents were relatively engaged consciously in filling the questionnaire. In testing for multi-collinearity, collinearity analyses revealed that tolerance values (0.20-0.94) lager than 0.10 and variance inflation (1.05-5.03) smaller than 10 hence indicating no evidence of multi-collinearity among data. We found evidence of convergent validity because the variance extracted measures all exceed 60 percent level and the realiabilty estimated of the contructs are larger than 0.7 (see Table 1). Furthermore, there was evidence of divergent validity because the variance extracted estimates for each factor are larger than the squared inter-construct correlations associated factor

Table 2. Descriptive statistics on variables

\begin{tabular}{llllllll}
\hline Variable & Mean & Std. & \multicolumn{6}{l}{ Pearson correlation coefficient $(p)$} & & \\
& & Deviation & 1 & 2 & 3 & 4 & 5 \\
\hline MT & 4.2434 & .67715 & 1.000 & & & & \\
LR & 3.9159 & .74854 & .615 & 1.000 & & & \\
PCV & 3.6711 & .88079 & .686 & .515 & 1.000 & & \\
OPC & 2.9093 & 1.07681 & .132 & .152 & .245 & 1.000 & \\
PSE & 3.7891 & .68223 & .276 & .299 & .351 & .142 & 1.000 \\
\hline
\end{tabular}

Note: $\mathrm{MT}=$ Motivation to transfer, $\mathrm{LR}=$ Learning readiness, $\mathrm{PCV}=$ Perceived Content Validity, $\mathrm{PSE}=$ Personal Self-Efficacy, $\mathrm{OPC}=$ Organization openness to Change 


\subsection{Common Method Variance}

Because the data for this investigation were collected from a single source at one time, i.e. training participants' self-reports at immediate end of training, a Harmon one factor test was conducted to determine the extent of a potential common method bias in the data . Result suggested the presence of the five factors, thus indicating that the common method variance was unlikely to influence the observation in this study.

\subsection{Validation of the Regression Model}

The hypothesized model of transfer motivation predictors at the pre-training stage yielded an acceptable fit to data. In summary $\mathrm{R}=.567$; which means that taken as a set the predictors learning readiness, perceived content validity, personal capacity for transfer, performance self-efficacy, and organization open to change account for $56.7 \%$ of variance in motivation to transfer training at the work place. The overall model was significant $F(5,220)=57.5 \mathrm{p}<0.001$ indicating that, taken as a group the predictors learning readiness, perceived content validity, personal capacity for transfer, performance self-efficacy, and organization open to change predicts motivation to transfer training significantly.

Table 3. Multiple regression output

\begin{tabular}{llllc}
\hline DV : MT & Coefficient (b) & Std. Error & t stats(t) & p-value $(\boldsymbol{p})$ \\
\hline LR & .325 & .209 & 6.868 & .000 \\
PCV & .396 & .042 & 9.514 & .000 \\
PSE & -.005 & .048 & -.100 & .921 \\
OPC & -.030 & .029 & -1.049 & .295 \\
Constant & 1.652 & .209 & 7.792 & .000 \\
R-squared=0.566 & & & & $\mathrm{F}(5,220)=57.5$ \\
Adj.R-squared $=0.559$ & & & & Prob $>\mathrm{F}=0.000$ \\
\hline
\end{tabular}

Note: $\mathrm{MT}=$ Motivation to transfer, $\mathrm{LR}=$ Learning readiness, $\mathrm{PCV}=$ Perceived Content Validity, $\mathrm{PSE}=$ Personal Self-Efficacy, $\mathrm{OPC}=$ Organization openness to Change.

Looking at the predictors individually we can see from Table 3 that learning readiness is significant meaning that, the amount of unique variance learning readiness account for in motivation to transfer is significant. What we mean by unique is that the amount of variance that learning readiness account for explain something unique in motivation transfer that the other predictors did not. Also, since Perceived content validity and organization openness to change are also significant, this means that this predictor also explains an amount of unique variance in motivation to transfer at the work place. However, performance self-efficacy was not significant. 


\section{Discussion}

The starting point of this study was to investigate how the trainee answers before the training program to the questions "Can I do this Task?" "Do I want to do this task and why?" and the organization openness to change influence the motivation to transfer learning at the post-training stage. We hypnotized that pre-training self-efficacy, learning readiness, perceived content validity of the training program along with the organization openness to change account for significant variance in trainee motivation to transfer at the post-training stage. Data collected using LTSI questionnaire in Thailand from high and vocational school teachers participating in training on cloud computing integration in teaching reveal four major findings. Table 4 displays the results of the hypothesized independent variables.

Table 4. Coefficient

\begin{tabular}{lll}
\hline Learning readiness & Sig & $\mathrm{P}<0.001$ \\
\hline Perceived Content validity & $\mathrm{Sig}$ & $\mathrm{P}<0.001$ \\
Organization open to change & $\mathrm{Sig}$ & $\mathrm{p}=0.29$ \\
Performance self-efficacy & $\mathrm{Not}$ sig & $\mathrm{P}=0.899$ \\
\hline
\end{tabular}

$($ Test each predictor at alpha $=0.5)$.

First, learning readiness which is the extent to which individuals are prepared to enter and participate in training event is a significant predictor of motivation to transfer training (Hypothesis 2). As it was posited by Reid, Bates et al. (2007), learner readiness concept reveals trainee understanding of (a) how the training program affects the performance, (b) understanding about job related developments, (c) expectations from training, and (d) expected outcomes at the beginning of the training. This findings confirm previous research which have found that learning readiness is a predicator for motivation to transfer (Chang \& Chiang, 2013; Wenzel \& Cordery, 2014;Hutchins, Nimon, Bates, \& Holton, 2013). In order for the trainee to fully decide whether he or she can really apply the new knowledge or skill at the job, they need to be informed before the training about all the aspects of the training including objectives. Trainees and managers should communicate prior to training to establish a common goal of training. Managers must grasp difference in needs of trainees and fully comprehend the contents of training. The communication with trainees should follow a certain procedure and paradigm and must be carried out in pre - training stage. Such a communication is called "the communication mechanism for pre - training consensus". Training practitioners should serve as consultants to help trainees to be better prepared for training. Once the trainees are aware and comprehend the aim and objective on the training program and most importantly when they have participated in framing these objectives. They come to the training ready to learn and since the objectives of the training correspond to their currents challenges, they will be motivated to transfer the skills acquired in training.

For the specific case of teachers in Thailand, this finding is in line with the report given by 


\section{Macrothink}

Atagi (2014). In his findings, 96\% of Thai's teachers are trained in subjects they consider not to be a priority. Suggesting that majority of training are done without need assessment thus explaining the lack in motivation to transfer. New technology such as cloud computing requires prerequisite knowledge, skills, attitude, and a level of preparedness here referred as learning readiness. When teachers understand the role, importance, and expectation of integrating such an innovation, they will be actively motivated to transfer the skill and knowledge in to the job.

The second main finding revealed in this study concerns the low direct effect of pre-training performance self-efficacy on the motivation to transfer (Hypothesis 1). Contrary to our expectation which assumes that the pre-training self-efficacy is the most important characteristic that determines a person's motivation to transfer given the fact that, expected outcomes are filtered through perceptions of being able to perform the behavior in the first place. Results show that performance self-efficacy is only marginally but not significantly affecting motivation to transfer.

In training transfer research, many studies have observed the impact of self-efficacy on transfer. Researchers have found that self-efficacy is positively related to transfer of training (Blume et al., 2010). Individuals' confidence in their own ability to use newly acquired knowledge or skills affects the way in which they will apply them in the workplace. However, in both conceptual and operational terms, the relationship between pre-training self-efficacy and motivation to transfer has not been investigated thoroughly. Research exploring employees' reasons for applying training on the job has operated within social cognitive theory. Of the different scales used (learning confidence, computer confidence, general and performance self-efficacy) the strongest factor for predicting transfer motivation has been found in post-training performance self-efficacy rather than pre-training performance self-efficacy (Bell et al., 2017). Given the fact that some of our data were collected the very first day of the training activities, trainees without necessarily making the difference in their performance self -efficacy before the training event and after, explain why pre-training performance self-efficacy seems to be low relevant for transfer motivation.

Third, our finding supports the fact that, when trainees are able to provide an answer to the questions "do I want to do this task and why?" at the pre-training stage, they will have high motivation to transfer training at the post-training stage. Perceived content validity of the training content was observed to account for significance of variance in the motivation to transfer (Hypothesis 3). This results are consistent with result of previous studies (Ruona et al., 2002; Yamnill \& Mclean, 2005). Perceived Content validity at the pre-training stage set the tone of interest for the participants and influence their motivation to transfer. Perceived content validity of the training is the most important factor that affects transfer of training in Thailand, learners should be assigned as full stakeholders in designing and implementing training. Learners may be responsible for identifying training objectives, assessing their training needs, and participating in developing the training curriculum. The relevance of knowledge, skills, and attitudes of learners to training is significant in determining training transfer. 


\section{MInstitute Macrothin}

Finally, the organization openness to change was significantly link to the variance in motivation to transfer (Hypothesis 4). This result is in line with previous finding which have reported that an organizational learning culture reflecting values and beliefs about the importance of learning at work is positively related to trainees' transfer motivation (Reid Bates \& Khasawneh, 2005; Ruona et al., 2002). The perception of the organization openness to change by the trainee is build up from previous training experience. Trainees who have experienced successfully implement of skill or knowledge acquired from training before, have a positive view of the organization openness to change. They are therefore more motivated to try new skill learn in training.

\section{Conclusion}

Motivation to transfer is essential for the transfer of training from the workshop to the workplace. Without motivation, newly acquired knowledge and skills will not be applied at work. In organisational contexts, where positive transfer of training is generally regarded as the paramount concern of training efforts, new perspective have emerge focusing on the pre-training factors as they influence post-training outcomes.

This study contribute to general training planning strategy where managers and trainers should pay attention to trainee pre-training learning characteristics including their learning readiness, perceived content validity and perceived organization openness to change. Particularly, as Thailand education institution engage into the adoption of cloud computing tools in teaching on the perspective of "smart Thailand 2020", exploring teachers characteristics at the pre-training stage through the questions "can I do this task ? "Do I want to do this task and why?" will provide elements that fuel up the motivation to transfer at the post-training stage in term of choice, effort, and persistence in implementation.

\section{Limitations}

Some limitation might be related to collecting data. Potential shortcoming in the study is common method bias. We used one single questionnaire to measure all constructs included, so perhaps the strength of the association between these constructs may be somewhat inflated. Also, with respect to teaching discipline, this study did not distinguish teachers according to the subject they teach. Therefore, we encourage scholars and practitioners to narrow down the teachers' learner characteristics for cloud computing according to the discipline taught and compere to see if there is any difference in the relationship between their learning characteristics and motivation to transfer.

\section{Acknowledgement}

The research was supported by English for Intergraded Study (EIS) Association Thailand. Thanks to Mr.Surapong Ngamsom. President of EIS Association Thailand.

\section{References}

Appova, A., \& Arbaugh, F. (2017). Teachers??? motivation to learn: implications for supporting professional growth. Professional Development in Education, 1-17. https://doi.org/10.1080/19415257.2017.1280524 
Atagi, R. (2014). Policy Research in Asia Secondary Teachers in Thailand.

Bandura, A. (2009). Social Cognitive Theory of Mass Communication Social Cognitive Theory of Mass Communication, (October 2011), 37-41. https://doi.org/10.1207/S1532785XMEP0303

Banerjee, P., Gupta, R., \& Bates, R. (2017). Influence of Organizational Learning Culture on Knowledge Worker's Motivation to Transfer Training: Testing Moderating Effects of Learning Transfer Climate. Current Psychology, 36(3), 606-617. https://doi.org/10.1007/s12144-016-9449-8

Bates, R., Holton, E. F., \& Paul, J. (2012). A revised learning transfer system inventory: factorial replication and validation, 15(5), 549-569.

Bates, R., \& Khasawneh, S. (2005). Organizational learning culture, learning transfer climate and perceived innovation in Jordanian organizations. International Journal of Training and Development, 9(2), 96-109. https://doi.org/10.1111/j.1468-2419.2005.00224.x

Bell, B. S., Tannenbaum, S. I., Ford, J. K., Noe, R. A., \& Kraiger, K. (2017). 100 years of training and development research: What we know and where we should go. Journal of Applied Psychology, 102(3), 305-323. https://doi.org/10.1037/apl0000142

Blume, B. D., Ford, J. K., Baldwin, T. T., Huang, J. L., Ford, J. K., \& Baldwin, T. T. (2010). Transfer of Training: A Meta-Analytic Review, (December 2009). https://doi.org/10.1177/0149206309352880

Bozdoğan, D., \& Özen, R. (2014). Use of ICT technologies and factors affecting pre-service ELT teachers' perceived ICT self-efficacy. Turkish Online Journal of Educational Technology, 13(2), 186-196.

Broussard, S. C. (2004). The Relationship Between Classroom Motivation and Academic Achievement in Elementary-School-Aged Children. Family and Consumer Sciences Research Journal, 33(2), 106-120. https://doi.org/10.1177/1077727X04269573

Chang, J., \& Chiang, T. (2013). The Impact of Learner Characteristics on Transfer of Training. Www.jitae.org Journal of Information Technology and Application in Education (JITAE), 2(1), 16-22.

Chiaburu, D. S., \& Marinova, S. V. (2005). What predicts skill transfer? An exploratory study of goal orientation, training self-efficacy and organizational supports. International Journal of Training and Development, 110-123. https://doi.org/10.1111/j.1468-2419.2005.00225.x

Chung, Y. (2013). Trainee Readiness For Diversity Training. Journal of Diversity Management (Online), 8(2), 77-n/a. https://doi.org/10.19030/jdm.v8i2.8234

De Rijdt, C., Stes, A., van der Vleuten, C., \& Dochy, F. (2013). Influencing variables and moderators of transfer of learning to the workplace within the area of staff development in higher education: Research review. Educational Research Review, 8, 48-74. 
https://doi.org/10.1016/j.edurev.2012.05.007

Devos, C., Dumay, X., Bonami, M., Bates, R., Iii, E. H., Learning, T., ... Ltsi, I. (2007). The Learning Transfer System Inventory ( LTSI ) translated into French : internal structure and predictive validity. International Journal of Training and Development, 11(3), 181-199.

Edwards, J. S. (2013). Factors affecting training transfer in supervisors and hourly employees in a manufacturing organization, 221.

Forehand, J. W., Miller, B., \& Carter, H. (2017). Integrating Mobile Devices Into the Nursing Classroom. Teaching and Learning in Nursing, 12(1), 50-52. https://doi.org/10.1016/j.teln.2016.09.008

Gegenfurtner, A., Veermans, K., Festner, D., \& Gruber, H. (2009). Integrative Literature Review: Motivation to Transfer Training: An Integrative Literature Review. Human Resource Development Review, 8(3), 403-423. https://doi.org/10.1177/1534484309335970

Grohmann, A., \& Kauffeld, S. (2013). Evaluating training programs: development and correlates of the Questionnaire for Professional Training Evaluation. https://doi.org/10.1111/ijtd.12005

Han, J., Yin, H., \& Boylan, M. (2016). Teacher motivation: Definition, research development and implications for teachers. Cogent Education, 3(1), 1217819. https://doi.org/10.1080/2331186X.2016.1217819

Hutchins, H. M., Nimon, K., Bates, R., \& Holton, E. (2013). Can the LTSI Predict Transfer Performance? Testing intent to transfer as a proximal transfer of training outcome. International Journal of Selection and Assessment, 2(3). https://doi.org/10.1111/ijsa.12035

Iftakhar, S. (2016). Google classroom: what works and how? 3, 12-18.

Iii, E. F. H., Bates, R. A., Bookter, A. I., \& Yamkovenko, V. B. (1996). Convergent and Divergent Validity of the Learning Transfer System Inventory, 18(3). https://doi.org/10.1002/hrdq

Kao, C., Tsai, C., \& Shih, M. (2014). International Forum of Educational Technology \& Society Development of a Survey to Measure Self-efficacy and Attitudes toward Web-based Professional Development among Elementary School Teachers Development of a Survey to Measure Self-efficacy and Attitude. Journal of Educational Technology \& Society, 17(4), 302-315.

Kirwan, C., \& Birchall, D. (2006). Transfer of learning from management development programmes: Testing the Holton model. International Journal of Training and Development, 10(4), 252-268. https://doi.org/10.1111/j.1468-2419.2006.00259.x

Kontoghiorghes, C. (2002). Predicting Motivation to Learn and Motivation to Transfer Learning Back to the Job in a Service Organization: A New Systemic Model for Training Effectiveness. Performance Improvement Quarterly, 15(3), 114-129. https://doi.org/10.1111/j.1937-8327.2002.tb00259.x 


\section{Macrothink}

Human Resource Research

ISSN 1948-5441 2018, Vol. 2, No. 1

Korthagen, F. (2017). Inconvenient truths about teacher learning: towards professional development 3.0. Teachers and Teaching: Theory and Practice, 23(4), 387-405. https://doi.org/10.1080/13540602.2016.1211523

Maung, K. M., \& Chemripong, S. (2014). The Impact of Feedback on Transfer of Training. International Business Management, 8(6), 357-360.

Noe, R. A., \& Schmitt, N. (1986). The Influence of trainee attitudes on training effectiveness: Test of a model. Personnel Psychology, 39(3), 497-523. https://doi.org/10.1111/j.1744-6570.1986.tb00950.x

Prasertsilp, P., \& Olfman, L. (2014). Effective Teacher Training for Tablet Integration in K-12 Classrooms. 2014 47th Hawaii International Conference on System Science Effective, (1), 52-61. https://doi.org/10.1109/HICSS.2014.16

Rotermund, S., Deroche, J., Ottem, R., Owens, C., \& O 'rear, I. (2017). Teacher Professional Development By Selected Teacher and School Characteristics: 2011-12. Stats In Brief, (5), $1-21$.

Ruona, W. E. A., Iii, E. F. H., \& Bates, R. (2002). The relationship between learner utility reactions and predicted learning transfer among trainees, 218-228.

Wenzel, R., \& Cordery, J. (2014). Training Transfer Research: A Manager's Guide and Bibliography. Retrieved from http://www.aimwa.com/ /media/Advocacy/Research/Training Transfer Research A Managers Guide and Bibliography.ashx

Yamnill, S., \& Mclean, G. N. (2005). Factors Affecting Transfer of Training in Thailand. Human Resource Development Quarterly, 16(3), 323-343. https://doi.org/10.1002/hrdq.1142

Zubairy, N. F. A. A., Mozie, N. M., \& Ghazali, N. (2015). Work environment and training transfer: The moderating effects of motivation. In Proceedings of 2014 2nd International Conference on Technology, Informatics, Management, Engineering and Environment, TIME-E 2014 (pp. 194-199). https://doi.org/10.1109/TIME-E.2014.7011617

\section{Copyright Disclaimer}

Copyright for this article is retained by the author(s), with first publication rights granted to the journal.

This is an open-access article distributed under the terms and conditions of the Creative Commons Attribution license (http://creativecommons.org/licenses/by/3.0/). 